\title{
REVIEW
}

\section{Germline cell formation in Drosophila embryogenesis}

\author{
Masukichi Okada* \\ 2-5-10 Kohoku, Tsukuba, Ibaraki 300-0032, Japan
}

\begin{abstract}
The classical term germ cell determinant was defined to cover whole function of germ plasm, or morphologically remarkable cytoplasm localized in oocytes and germ cells. Since early 1970 consequential advances in technology, including microsurgery, genetic anatomy and gene manipulation, have allowed cytological and molecular analyses of pole plasm (germ plasm) in Drosophila embryos. A series of recent work has revealed that multiple factors or molecule species in pole plasm participate in a consecutive reactions resulting in germline formation. Two molecule species have been specified as playing roles in germline cell formation. One that participates in sequestering pole plasm from somatic environment is mitochondrial large ribosomal (mtlr) RNA, and the other involved in driving pole cells into the germline pathway is Nanos protein. Studies disclosed the roles of those two molecules are reviewed.
\end{abstract}

An individual multicellular animal comprises two types of cells, mortal somatic cells and immortal germ cells. In the embryonic development, somatic cells differentiate to produce various cell types. While each cell type has a distinct function, all somatic cells cooperate to settle an appropriate condition for germ cells being loaded with a complete set of DNA representing the individual and released safely out of this somatic body. Germ cells are thus regarded as vehicles for DNA to be delivered to the next generation (cf., Clark, 1996). Although germ cells undergo differentiation into sperms or eggs, their somatic differentiation is avoided for protecting germinal DNA from any possible modification or damage. In addition, for the same purpose, they are segregated from somatic cells at very early developmental stages in some animal groups.

Germ cells are recognizable as morphologically remarkable cells at early stages of embryonic development from early cleavage (e.g., Caenorhabditis elegans; Strome and Wood, 1983) to early gastrulation (e.g., chicken; Nieuwkoop and Sutasurya, 1979). In the fruit fly Drosophila melanogaster progenitor cells of germline are produced in the posterior region of preblastoderms, several minutes ahead of nuclear arrival at other (somatic) regions.

Observations on wide spread animal groups allow us to discriminate two classes of animals, one with and one without germ plasm. Germ plasm is localized in a particular region of the oocyte, sequestered from "somatic" cytoplasm during embryogenesis to be exclusively distributed to a fraction of cells, and influences these cells to follow the path-

\footnotetext{
* Professor Emeritus, University of Tsukuba, Tsukuba, Ibaraki 305-0006, Japan.
}

way to determination and differentiation as germ cells. In some insect species, only nuclei surrounded by germ plasm can escape from chromosome elimination that limits the cell fate to the somatic line (Beams and Kessel, 1974). In contrast, in animals without germ plasm, germ cells make their appearance later in embryogenesis. In those animals germ cells seem to diverge from a group of cells that have once followed the somatic pathway (Eddy et al., 1981).

In Drosophila, as in other dipteran insects, germline precursor cells or pole cells are distinct at the syncytial blastoderm stage (Sonnenblick, 1950). Nuclei taken from the anterior of the syncytial blastoderm are able to contribute to germline formation when transplanted into the posterior of early cleavage embryos (Okada et al., 1974b). This simply supports the classical concept of germ cell determination by the posterior pole cytoplasm that includes germ plasm. Posterior pole cytoplasm of the Drosophila embryo is referred to as pole plasm.

As UV irradiation of pole plasm deprived of germ cellforming ability from the embryo, pole plasm was suggested to include germ cell determinant (Aboim, 1945). However, further understanding of pole plasm for its biological function and molecular anatomy had to wait until technology in experimental embryology, genetics, and molecular biology became available in Drosophila.

\section{Pole Cells: Morphological and Genetical Character- istics}

Determination of primordal germ cells (PGCs) as the germline is not strict in some animal groups. Actually, in amphibian embryos PGCs differentiate along with surrounding cells where the PGCs are transplanted (Wylie 
et al., 1985). In contrast, pole cells (prospective PGCs in Drosophila) have no ability to differentiate as somatic cells even when transplanted into a given somatic region of a blastoderm (Technau and Campos-Ortega, 1986). Thus pole cells have definitely been following the pathway to develop to germline since they are formed, and never be able to proceed the way back under conditions so far tested.

Pole cells have distinguishable morphological characteristics. They are round in profile, and each has nucleus including electron dense nuclear bodies (Mahowald, 1962).

It was reported in Drosophila that germline cells in adult gonads had a unique ability to splice the third intron out of P element transcripts (Laski et al., 1986). To examine if pole cells have a similar ability, a $\mathrm{P}$ element with an $h s p 70$ promoter and with $l a c Z$ gene inserted in its exon 4 was introduced into a Drosophila strain by the P element mediated transformation method (Spradling and Rubin, 1982). LacZ translates are generated in the transformant only when the third intron is removed from the hybrid $\mathrm{P}$ element transcripts. This survey gave a clear result that pole cells acquire the splicing ability when they reach the posterior midgut rudiment in the proctodaeal invagination (Fig 1). Surprisingly, however, not all pole cells in an transformed embryo share this ability. It was approximately $10 \%$ of pole cells that had this ability. Further observations suggest that only pole cells with this splicing activity survive gametogenesis to differentiate as germ cells (Kobayashi et al.,1993).

These findings raise the possibility that only a fraction of pole cells incorporates sufficient amount of factors responsible for pole cell differentiation as germline. Molecular basis tracking of the factors will be needed to test this possibility.

\section{Pole Cell Formation}

\section{A) Pole plasm}

i) Morphology. Around 10 nuclei among 258 generated after the 8th cleavage migrate posteriorly ahead of the other nuclei to reach pole plasm, where are formed protrusions each including a nucleus and a fraction of pole plasm, finally to become pole cells (Fig. 1). It is essential for cleavage nuclei to be involved in pole plasm for their contribution to germline establishment. Germ plasm can be described as morphologically remarkable cytoplasm localized in oocytes being inherited to germline. For its remarkable morphological features, germ plasm has been reported to exist in various animal groups covering seven phyla (Eddy, 1975). Apparently, however, germ plasm or pole plasm has most thoroughly been documented in $D$. melanogaster.

Polar granules were noted with classical light microscopy as minute granules exclusively present in pole plasm and later included in germline (for review, see Counce, 1973). The fine structure of these granules was described in detail with electron microscopy, as non-membrane limited electron dense bodies, including granular and fibrous com-

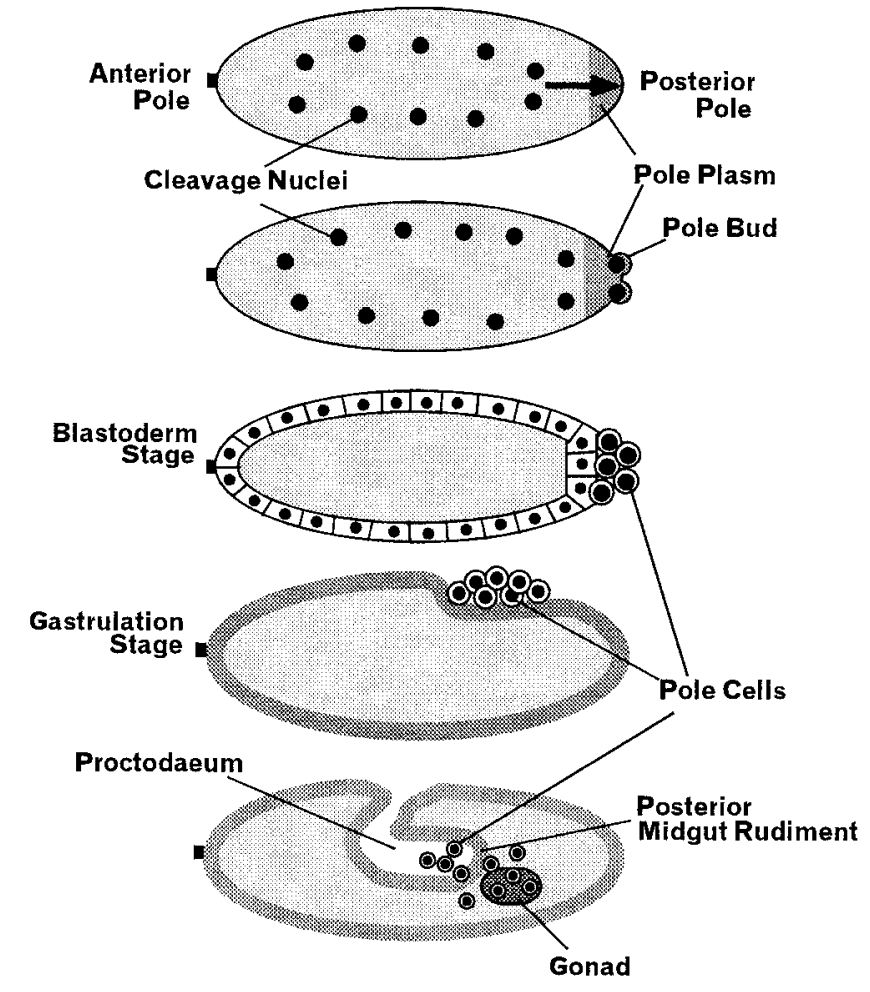

Fig. 1. Five schematic drawings of embryos of increasing stages to illustrate production of pole cells and their migration to reach embryonic gonad.

ponents (Mahowald, 1962). Furthermore, polar granules have been continuously tracked through the life cycle of Drosophila, only missing in a narrow window in oogenesis when nurse cells synthesize new pole plasm components for replacing old ones (Mahowald, 1971). The contour of polar granules in thin sections for electron microscopy is variable but characteristic of each species of genus Drosophila (Mahowald, 1968).

ii) Experimental analyses. As mentioned above UV sensitivity of pole plasm in its germline determining activity was noticed earlier. Further studies revealed that irradiation of Drosophila early cleavage embryos posteriorly with $280 \mathrm{~nm} \mathrm{UV}$ at $200 \mathrm{~J} / \mathrm{m}^{2}$ gave the most efficient sterility with the least damage to somatic cell formation (Togashi and Okada, 1983). UV-irradiated embryos restored fertility if they were transplanted immediately after the irradiation with pole plasm taken from non-irradiated embryos. No cytoplasm other than pole plasm had ability to restore fertility to UV-irradiated embryos (Okada et al., 1974b). If an early cleavage embryo was transplanted with pole plasm at an ectopic region, pole cell-like round cells were formed at the transplanted site (Okada et al., 1974b). Those ectopic pole cells were neatly demonstrated to be capable of differentiating as germline cells (Illmensee and Mahowald, 1974). These experiments thus confirmed the classical belief that pole plasm includes germ cell determinant.

iii) Mutations with grandchildless phenotype. Since 
pole plasm components are produced in nurse cells under the control of maternal genes and transported to localize in the oocyte, some maternal effect mutations cause failure in pole cell formation. The mutation is termed grandchildless, because the first generation is normally produced from mutant females but no second generation arises as the result of deficiency in pole cells in embryogenesis of the first generation. First grandchildless (gs) mutant was reported from D. subobscura (Spurway, 1948). Although gs mutants were artificially generated and screened later in $D$. melanogaster, they were found to give no direct effect to pole plasm (for review, see Konrad et al., 1985). Actually, the normal autonomous function was demonstrated in pole plasm from embryos produced by females with a grandchildless mutation (gs(1)N26) (Niki, 1984).

On the other hand, it was noticed that a mutation at any locus of posterior group genes results in absence of pole cells, in addition to aberrant abdominal segments. Posterior group genes are vasa (Schüpbach and Wieschaus, 1989), valois (Schüpbach and Wieschaus, 1989), staufen (Schüpbach and Wieschaus, 1989), tudor (Boswell and Mahowald, 1985), oskar (Lehmann and Nüsslein-Volhard, 1986), cappuccino (Manseau and Schüpbach, 1989), spire (Manseau and Schüpbach, 1989), mago nashi (Boswell et al., 1991), pumilio (Nüsslein-Volhard et al., 1987) and nanos (Lehmann and Nüsslein-Volhard, 1991). Among them pumilio and nanos seem only to affect abdominal segmentation. Pole cells are formed in embryos produced by females with pumilio or nanos mutation. However, others produce embryos deficient in pole cells as well as in abdominal segments.

\section{B) Necessity of nuclei in pole cell formation}

Pole cell formation starts at the arrival of nuclei in pole plasm. However, nuclear arrival at pole plasm seems not to be sufficient for initiating pole cell formation. Maternal effect mutations, gs(1)N26 and gs(1)N441 were isolated as ones with grandchildless phenotype (Niki and Okada, 1981). The defect caused by these mutations is not in pole plasm (Niki, 1984), but in nuclear migration. In fact nuclear arrival in pole plasm is delayed in mutant embryos by 30 minutes or more from the normal procedure (Niki and Okada, 1981; Hatanaka and Okada, 1991). As temperature sensitive are those mutations, nuclear arrival at the posterior is not late at a permissive temperature. In those embryos pole cells are normally formed.

To test correlation between delay in nuclear arrival to the posterior and deficiency in pole cell formation, posteriorward migration of nuclei was artificially prevented by partial ligation of embryos to make nuclear penetration into pole plasm delayed by various period of time. It was found that no pole cells were formed when nuclei failed to penetrate pole plasm within 30 minutes after the normally scheduled time of nuclear arrival in pole plasm. The disorder caused by delay in nuclear arrival was canceled with transplantation of polar plasm taken from embryos at an earlier stage (Okada, 1982, 1986). The results propose the possibility that pole plasm may have a critical period of time for starting pole cell formation responding to nuclei. Experiments to replace normal pole plasm by "aged" pole plasm have to be done, and molecular analyses are also needed, for proper explanation of this phenomenon.

Raff and Glover (1989) reported that aphidicolin injected into Drosophila embryos at nuclear cycle 7-8 inhibited nuclear migration, and this agent also causes centrosomes to be separated from nuclei. In these embryos, centrosomes continued to migrate, and penetrated pole plasm where pole cells containing only centrosomes were formed. These observations demonstrate that centrosomes alone are able to reorganize cortical cytoskeleton to initiate pole cell formation.

\section{C) Pole cell-forming factors}

i) mtlrRNA as a pole cell-forming factor. Combination of UV irradiation and microinjection provides a good bioassay system for detecting factor(s) responsible for pole cell formation, although there is a limitation that only UVsensitive factors are detectable (Fig. 2). Relying on this assay system, factors were traced in a subcellular fraction (Ueda and Okada, 1982), then in poly $(\mathrm{A})^{+} \mathrm{RNA}$ (Togashi et al., 1986). Poly(A) RNA extracted from early cleavage embryos but not that from blastoderms was active in rescuing UV-irradiated embryos from sterility. Using this stage specificity for selection, cDNA library was generated from early cleavage embryos and screened for cDNA coding RNA with pole cell-forming activity. To our surprise, cDNA we screened exhibited very high (98\%) identity with the mitochondrial large ribosomal RNA (mtlrRNA) gene of $D$. yakuba in a data base. No nuclear gene was homologous with the cDNA we screened. RNA generated in vitro from full length cDNA was highly active in rescuing UV-irradiated embryos for pole cell-forming ability (Kobayashi and Okada, 1989; Kobayashi and Okada, 1990).

As a component of the mitochondrial ribosome, mtlrRNA is present in mitochondria. However, our in situ hybridization to whole embryos detected mtlrRNA signals only in pole plasm of early cleavage embryos (Fig. 3). In situ hybridization technique was applied for electron microscopy, and it was demonstrated that mtlrRNA in pole plasm is present outside mitochondria and localized on polar granules (Fig. 4) (Kobayashi et al., 1993; Amikura et al., 1996). This observation has given an important piece of knowledge that mtlrRNA is a component of polar granules. Further electron microscopic observations suggest that transportation of mtlrRNA out of mitochondria takes place at the site where polar granules attaching, and this starts at egg laying, probably at fertilization (Amikura et al., 1996).

Presence of outside mitochondrial mtlrRNA (referred to as om-mtlrRNA, hereafter) has strong correlation with pole cell formation (Kobayashi et al., 1993). UV irradiation 


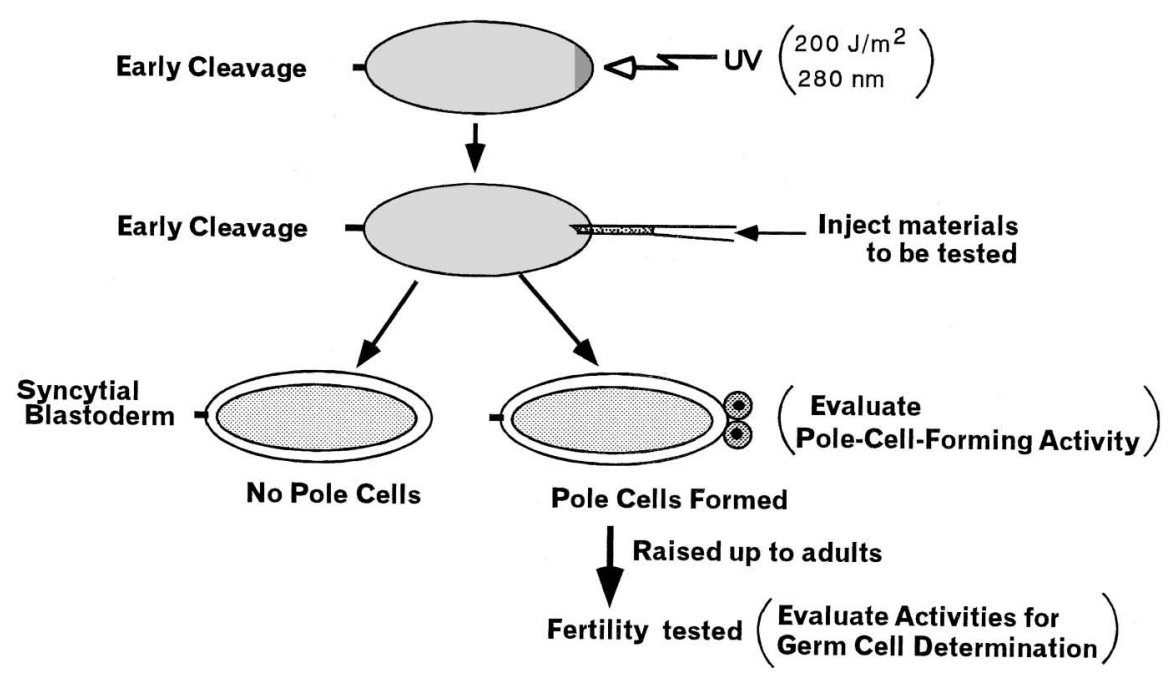

Fig. 2. Bioassay used for detecting UV-sensitive pole-cell-forming factors. When necessary, hatched larvae were collected and allowed to develop to adulthood.

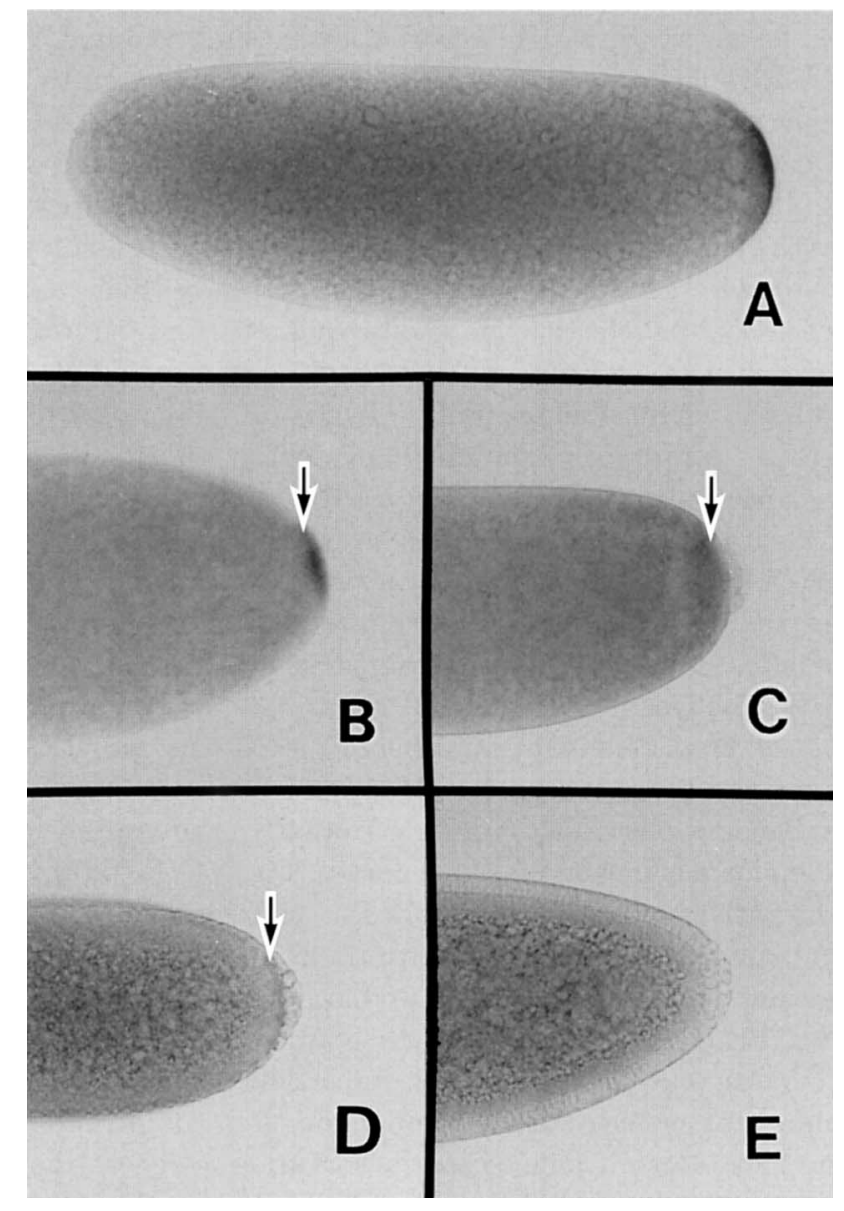

Fig. 3. Whole-mount in situ hybridization of mtlrRNA probes to embryos at the early cleavage (A), late cleavage (B), pole but (C), syncytial blastoderm (D), and cellular blastoderm stages (E). Arrows in B, C, and D point to mtlrRNA signal. Note that mtlrRNA is not included in pole cells, and disappears by pole cell formation is completed. (after Amikura et al., 1996) causes a definite decrease or deficiency of om-mtlrRNA in pole plasm. Bicaudal-D mutant embryos, whose anterior forms abdomen but no pole cells, has om-mtlrRNA only in the posterior pole plasm. In embryos with mutation at any posterior group loci, except nanos and pumilio, no ommtlrRNA is detected in pole plasm (Kobayashi et al., 1993; Ding et al., 1994).

Oskar mRNA is ectopically localized in the anterior pole of embryos, if their mother fly has been transformed with an oskar gene in which bicoid gene 3'UTR that includes a signal for anterior localization has been substituted for its original 3'UTR (osk-bcd 3'UTR) (Ephrussi and Lehmann, 1992). In these embryos, where osk mRNA localizes both in the anterior and posterior, localization of Vasa protein, Tudor protein and nanos mRNA as well as polar granule assembly is observed at both poles. In osk-bcd 3'UTR embryos, consequently, pole cells and abdomens are formed at both poles. Ding et al. (1994) reported that mtlrRNA was not concentrated in the anterior of $o s k$-bcd 3'UTR embryos. However, their observations were thoroughly repeated in more precisely designed series of experiments, and it was definitely demonstrated that om-mtlrRNA localized at both poles in osk-bcd 3'UTR embryos (Kobayashi et al., 1995). Taken together, transport out of mitochondria, and localization in pole plasm of om-mtlrRNA are regulated with a mechanism under the control of osk, vas, and tud, with osk at the highest in hierarchy.

Among the posterior group genes, tudor is particular. Tudor protein is reported to be present in mitochondria as well as in polar granules and surrounding cytoplasm in pole plasm (Bardsley et al., 1993). This proposes the possible role of Tudor protein mediating mtlrRNA to travel through mitochondrial membranes. Actually there is an observation suggesting that mtlrRNA co-exists with Tud protein all through the process of its migration from mitochondria 

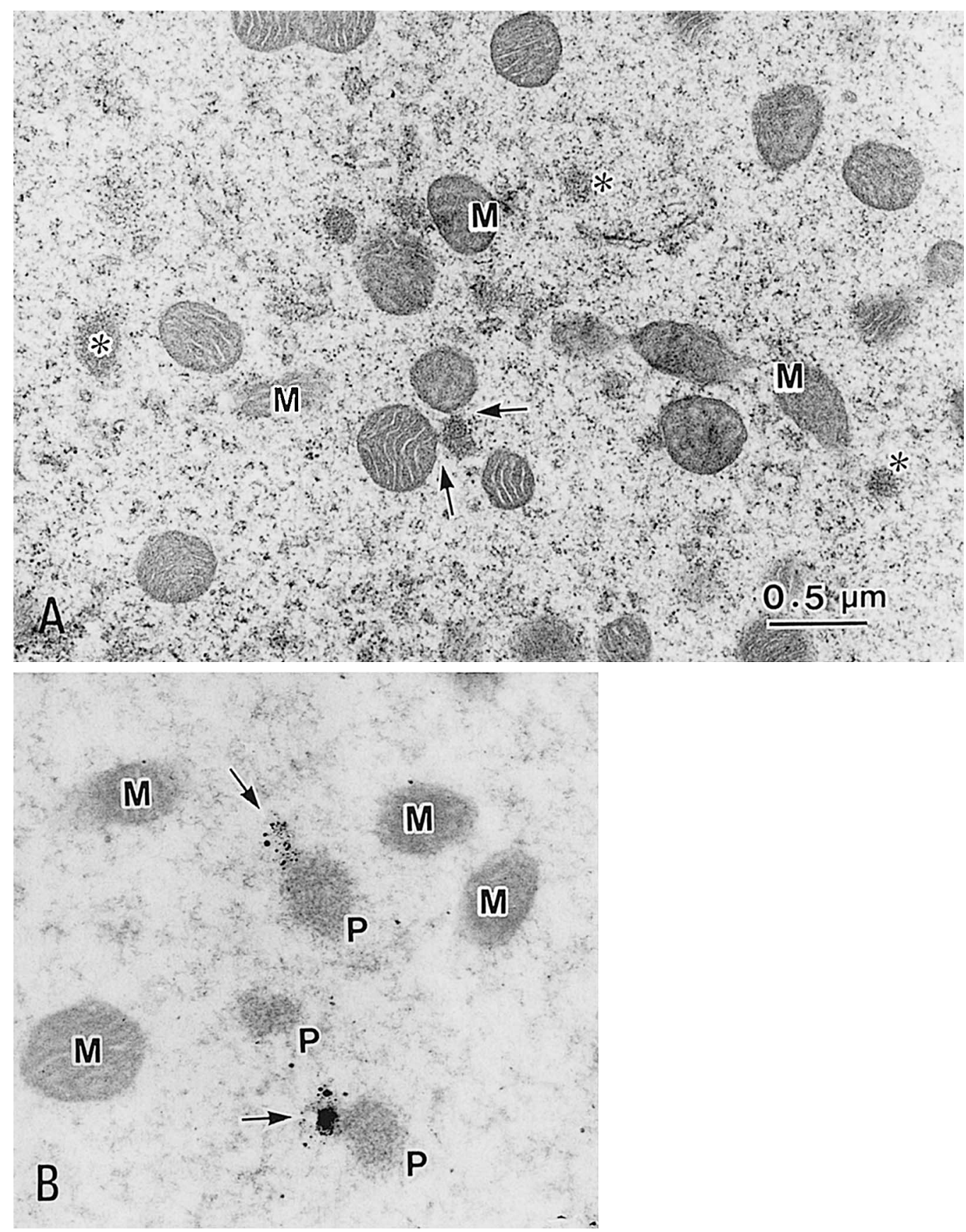

Fig. 4. Electron micrographs of sections through pole plasm of early cleavage embryos. A, Pole plasm of an embryo processed for a standard histology. B, pole plasm in which om-mtlrRNA is visualized with gold particles coupled to hybridization probes. M, Mitochondrion (A and B); p, Polar granules (B). Arrows in A indicate the site of contact between a mitochondrion and a polar granule. Arrows in B point to mtlrRNA signal. Asterisks in A show polar granules. (Courtesy of Dr. R. Amikura)

to polar granules (Amikura et al., 1997).

Recently, ribozymes targeting specifically at either of several selected loci of mtlrRNA were generated and injected into pole plasm of wild-type embryos at an early cleavage stage. Besides om-mtlrRNA signals significantly decreased in injected embryos, some of the ribozyme species prohibited pole cell formation, but some others did not (Iida and Kobayashi, 1997). Probably mtlrRNA has crucial sites in itself to its function in pole cell formation. Those lines of evidence offer a direct support to the idea that mtlrRNA is essential for pole cell formation. In this connection, injection of mtlrRNA was considered not simply to recover mitochondrial activity from UV damage, because UV irradiation did not affect the high level of mitochondrial respiratory activities in pole plasm (Akiyama and Okada, 1992). ii) The role of mtlrRNA. It has been demonstrated 
that mtlrRNA is a pole cell-forming factor associated with polar granules. Here I ask, to what extent it is involved in germline establishment in normal development (Fig. 5).

When injected into anterior pole of early cleavage embryos, mtlrRNA alone did not induce ectopic pole cell. When coinjected with UV-irradiated pole plasm, mtlrRNA can induce pole cells at the anterior pole (Kobayashi et al., 1993). Needless to say, UV-irradiated pole plasm alone never induce ectopic pole cells. This observation suggests the possibility that another pole cell-forming factor, which is UV-resistant and cooperative in function with ommtlrRNA, exists in pole plasm.

On the other hand, pole cells produced in UV-irradiated embryos injected with cytoplasmic fraction, $\operatorname{poly}(\mathrm{A})^{+} \mathrm{RNA}$, or mtlrRNA, are not able to complete their proceeding through the pathway to germ cell differentiation (Ueda and Okada, 1982; Togashi et al., 1986; Kobayashi and Okada, 1989). Those pole cells are morphologically very similar, although not completely identical, to normal pole cells (Yamazaki and Okada, 1989), and are capable of composing gonads in cooperation with somatic mesodermal cells, where they themselves become PGCs. Nonetheless, those PGCs never develop to functional germ cells. The role of om-mtlrRNA should thus be limited to initiate pole cell formation. Some other factors may be required for directing pole cells to differentiate as germ cells. Recently, some candidates for those factors have been nominated and mists covering the whole process of germline formation is being dispelled. I will come back to this point later in the next section.

Cytological function of om-mtlrRNA in pole cell formation is still unknown. Since it is limited to pole cell formation, or protrusion from the cell surface and constriction to isolate round cells, it is rational to presume that om-mtlrRNA takes part in reorganization of cytoskeletons.
Yet, it is not known how it works. It was reported recently that in addition to mtlrRNA, mitochondrial small rRNA is transported out of mitochondria in pole plasm (Kashikawa et al., 30th JSDB Congress, 1997). One of their working hypothesis is that the prokaryote type protein synthesis on polar granules may be involved in pole cell formation. In this context, it may be worthwhile to repeat experiments in which no pole cell formation occurred when protein synthesis in pole plasm was inhibited by cycloheximide at early cleavage stages (most sensitive during 3rd and 4th cleavage) (Okada, 1986), with additional experiments using protein inhibitors for prokaryotes.

\section{Differentiation of Pole Cells as Germ Cells}

As mentioned above pole plasm of Drosophila embryos includes at least two pole cell-forming factors; om-mtlrRNA and a UV-resistant factor, to which germ cell-less products were suspected as a candidate (Kobayashi et al., 1995), and a factor(s) required for pole cells to develop to germ cells.

nanos mRNA is localized in pole plasm where translated to form a gradient of Nanos protein with its highest concentration in pole plasm. Nanos protein acts as a translational repressor for hunchback mRNA to direct abdomen formation (Lehmann and Nüsslein-Volhard, 1991). By the cellular blastoderm stage, Nanos protein disappears from the prospective abdomen region, but remains in pole cells through their migration and association with mesoderm cells to form embryonic gonads (Wang et al., 1994).

On speculation that these observations reflect an important role of Nanos protein in germline formation, pole cell transplantation was carried out to test whether pole cells produced in embryos from females with a nanos mutation were capable of developing to germ cells (Kobayashi et al., 1996). Their experiments clearly showed that nanos mutation affected pole cells in their migration to gonads re-

\section{Injection of mtIrRNA}

\section{into the anterior pole into the UV-irradiated posterior pole}

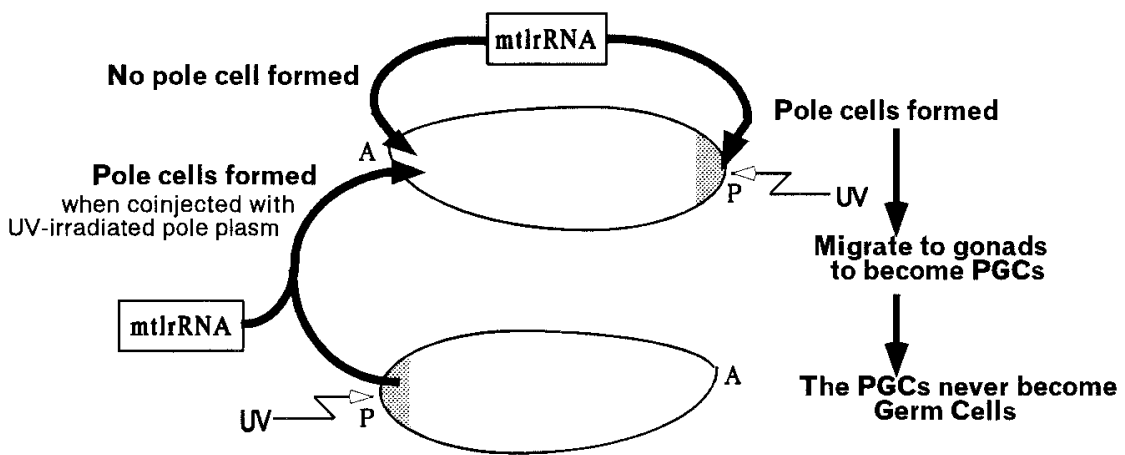

Fig. 5. Summary of injection of mtlrRNA into the anterior and posterior poles of early cleavage embryos. Into the anterior mtlrRNA was injected alone or coinjected with pole plasm that had been UV-irradiated. Injection at the UV-irradiated posterior pole provided two activities of mtlrRNA, pole cell formation and germ cell determination. 
sulting in their failure to differentiate as germ cells. In addition, it was demonstrated that those nanos pole cells performed premature synthesis of some proteins that takes place later in normal development. Apparently, Nanos protein contributes to germ cell differentiation with repressing the expression of a somatic gene(s) (Asaoka and Kobayashi, 1997). Pole cell migration to gonads is hampered if $p g c$ (polar granule component) gene function has maternally been disturbed with antisense pgc RNA. Pgc was identified as an untranslatable RNA, and found to be localized in polar granules (Nakamura et al., 1996).

Recently, a novel gene was reported to be expressed specifically in pole cells only when pole cells once accomplished association with mesoderm cells with a gonadal trend (Mukai et al., 1997). This implies that even after directed by maternal factors an external signal from cells in contact is important for pole cells to complete their development to germline.

\section{Conclusion}

According to Eddy (1975) polar granules were taken by Hegner (1914) in a coleopteran insect as visible evidence of specialized differentiation in the cytoplasm that controls the production of primordial germ cells, and were referred to as germline (Keimbahn) determinants. In amphibian eggs, Bounoure (1931) described morphologically remarkable cytoplasm. From further observations and experiments he concluded that this cytoplasm was related to germ cell differentiation and he named it cytoplasme germinal (Bounoure, 1939). The classical terms, germ plasm and germ cell determinant are thus considered to include a common concept of integrated function of cytoplasm to direct germ cell formation.

So far accumulated lines of evidence I presented in this review have revealed that the function of germ plasm or germ cell determinant is resolved at least into two phases. The components of pole plasm (germ plasm of Drosophila) have partially been specified. In the first phase, pole plasm is sequestered in pole cells. This process must involve reorganization of cytoskeleton that starts at nuclear arrival cueing om-mtlrRNA. As a matter of fact, however, not nuclei but centrosomes participate in this process. When pole plasm has been received into pole buds, or pole cell rudiments, om-mtlrRNA ceases its action to be decomposed without entering into pole cells. At the completion of the first phase, pole plasm is ensured to stay for good in pole cells. In the second phase, pole plasm components can thus be engaged exclusively in directing the pole cells to follow a single pathway to germ cell differentiation. Pole cell formation thus provides a closed and stable environment for maternally prepared pole plasm components to react among them and with nuclear genes. Actually Nanos protein remains much longer in pole cells than in somatic cytoplasm to take a crucial part in controlling pole cell development, although mechanism underlying persistence of Nanos pro- tein in pole cells is still elusive.

Reactions taking place in the second phase for directing germ cells to differentiate are presumably independent of the process in the first phase. However, packaging of pole plasm in a limited space of pole cells accomplished at the end of the first phase is essential for increasing efficiency and securing independence of reactions in the second phase. I assume that an inefficient reactions in the second phase appeared earlier in evolution, and the first phase was acquired later to increase efficiency of reactions. If I may be allowed to do a further assumption, multicellular organisms may have utilized a machinery ancient bacteria used for their particular cell division such as one in an emergency to form spores.

\section{REFERENCES}

Aboim, A. N. (1945) Développement embryonnair et postembryonnair des gonades normale et agametiques de Drosophila melanogaster. Rev. Suisse Zool. 52, 53-154.

Akiyama, T., and Okada, M. (1992) Spatial and developmental changes in the respiratory activity of mitochondria in early Drosophila embryos. Development 115, 1175-1182.

Amikura, R., Kobayashi, S., Saito, H., and Okada, M. (1996) Changes in subcellular localization of mtlrRNA outside mitochondria in oogenesis and early embryogenesis of Drosophila melanogaster. Dev. Growth Differ. 38, 489-498.

Amikura, R., Kashikawa, M., and Kobayashi, S. (1997) Localization of mitochondrial large ribosomal RNA and Tudor protein in early cleavage embryos of Drosophila melanogaster. Dev. Biol. 186, 305.

Asaoka, M., and Kobayashi, S. (1997) Nanos represses the expression of a somatic line marker in germline of Drosophila embryos. Dev. Biol. 186, 305.

Beams, H. W., and Kessel, R. G. (1974) The problem of germ cell determinants. Int. Rev. Cytol. 39, 413-479.

Boswell, R. E., and Mahowald, A. P. (1985) tudor, a gene required for assembly of the germ plasm in Drosophila melanogaster. Cell 43, 978-104.

Boswell, R. E. Prout, M. E., and Steichen J. C. (1991) Mutations in a newly identified Drosophila melanogaster gene, mago nashi, disrupt germ cell formation of mirror-image symmetrical double abdomen embryos. Development 113, 373-384.

Clark, W. R. (1996) Sex and the Origins of Death. Oxford Univ. Press, New York, Oxford.

Counce, S. J. (1973) The causal analysis of insect embryogenesis. In: Developmental Systems: Insects. vol. 2 (eds.: S. J. Counce, and C. H. Waddington), pp. 1-156. Academic Press, London and New York.

Ding, D., Whittaker, K. L., and Lipshitz, H. D. (1994) Mitochondrially encoded 16S ribosomal RNA is concentrated in the posterior polar plasm of early Drosophila embryos but is not required for pole cell formation. Dev. Biol. 163, 503-515.

Eddy, E. M. (1975) Germ plasm and the differentiation of the germ cell line. Int. Rev. Cytol. 43, 229-280.

Eddy, M. E., Clark, J. M., Gong, D., and Fenderson, B. A. (1981) Origin and migration of primordial germ cells in mammals. Gamete Res. 4, 333-362.

Hatanaka, K., and Okada, M. (1991) Retarded nuclear migration in Drosophila embryos with aberrant F-actin reorganization caused by maternal mutations and by cytochalasin treatment. Development 111, 909-920.

Iida, T., and Kobayashi, S. (1997) Role of mitochondrial large 
ribosomel RNA in pole cell formation of Drosophila. Zool. Sci. (Tokyo) 14 (suppl.) 69.

Illmensee, K., and Mahowald, A. P. (1974) Transplantation of posterior polar plasm in Drosophila. Induction of germ cells at the anterior pole of the egg. Proc. Natl. Acad. Sci. USA 71, 1016-1020.

Kobayashi, S., and Okada, M. (1989) Restoration of pole-cell-forming ability to u.v.-irradiated Drosophila embryos by injection of mitochondrial lrRNA. Development 107, 733-742.

Kobayashi, S., and Okada, M. (1990) Complete cDNA sequence encoding mitochondrial large ribosomal RNA of Drosophila melanogaster. Nucleic Acids Res. 18, 4592.

Kobayashi, S., Amikura, R., and Okada, M. (1993) Presence of mitochondrial large ribosomal RNA outside mitochondria in germ plasm of Drosophila melanogaster. Science 260, 15211524.

Kobayashi, S., Kitamura, S. T, Sasaki, H., and Okada, M. (1993) Two types of pole cells are present in the Drosophila embryo; one with and one without splicing activity for the third P-element intron. Development 117, 885-893.

Kobayashi, S., Yamada, M., Asaoka, M., and Kitamura, T. (1996) Essential role of the posterior morphogen nanos for germline development in Drosophila. Nature 380, 708-711.

Kobayashi, S., Amikura, R., Nakamura, A. Saito, H., and Okada, M. (1995) Mislocalization of oskar product in the anterior pole results in ectopic localization of mitochondrial large ribosomal RNA in Drosophila embryos. Dev. Biol. 169, 384-386.

Konrad, K. D., Engstrom, L., Perrimon, N., and Mahowald, A.P. (1985) Genetic analysis of oogenesis and the role of maternal gene expression in early development. In: Developmental Biology: A Comprehensive Synthesis, vol. 1. Oogenesis (ed.: L.W. Browder), pp. 577-617. Plenum Press, New York and London

Laski, F. A., Rio, D. C., and Rubin, G. M. (1986) Tissue specificity of Drosophila $\mathrm{P}$ element transposition is regulated at the level of mRNA splicing. Cell 44, 7-19.

Lehmann, R., and Nüsslein-Volhard C. (1986) Abdominal segmentation, pole cell formation, and embryonic polarity require the localized activity of oskar, a maternal gene in Drosophila. Cell 47, 141-152.

Lehmann, R., and Nüsslein-Volhard, C. (1991) The maternal gene nanos has a central role in posterior pattern formation of the Drosophila embryo. Development 112, 679-691.

Mahowald, A. P. (1962) Fine structure of pole cells and polar granules in Drosophila melanogaster. J. Exp. Zool. 151, 201-215.

Mahowald, A. P. (1968) Polar granules of Drosophila. II. Ultrastructural changes during early embryogenesis. J. Exp. Zool. 167, 237-262.

Mahowald, A. P. (1971) Polar granules of Drosophila. III. The continuity of polar granules during the life cycle of Drosophila. J. Exp. Zool. 176, 329-343.

Manseau L. J., and Schüpbach, T. (1989) cappuccino and spire: two unique maternal-effect loci required for both the anteroposterior and dorso-ventral patterns of the Drosophila embryo. Genes Dev. 3, 1437-1452.

Mukai, M., Kashikawa, M., and Kobayashi, S. (1997) A novel gene, \#098, expressed in pole cells within the embryonic gonads. Dev. Biol. 186, 305.

Nakamura, A., Amikura, R., Mukai, M., Kobayashi, S., and Lasko, P. F. (1996) Requirement for a noncoding RNA in Drosophila polar granules for germ cell establishment. Science 274, 2075-2079.

Nieuwkoop, P. D., and Sutasurya, L. A. (1979) Primordial Germ Cells in the Chordates. Cambridge Univ. Press, Cambridge.

Niki, Y. (1984) Developmental analysis of the grandchildless (gs(1)N26) mutation in Drosophila melanogaster: Abnormal cleavage patterns and defects in pole cell formation. Dev. Biol. 103, 182-189.

Niki, Y., and Okada, M. (1981) Isolation and characterization of grandchildless-like mutants in Drosophila melanogaster. Roux's Arch. Dev. Biol. 190, 1-10.

Nüsslein-Volhard, C. Frohnhöfer, H. G., and Lehmann, R. (1987) Determination of anteroposterior polarity in Drosophila. Science 238, 1675-1681.

Okada, M. (1982) Loss of the ability to form pole cells in Drosophila embryos with artificially delayed nuclear arrival at the posterior pole. In: Embryonic Development, Pt. A: Genetic Aspect (ed.: M. M. Burger and R. Weber), pp. 363-372. Alan R. Liss Inc., New York.

Okada, M. (1986) Cytoplasmic function segregating germline in Drosophila embryogenesis. Zool. Sci. (Tokyo) 3, 573-583.

Okada, M., Kleinman, I. A., and Schneiderman, H. A. (1974a) Restoration of fertility in sterilized Drosophila eggs by transplantation of polar cytoplasm. Dev. Biol. 37, 43-54.

Okada, M., Kleinman, I. A., and Schneiderman, H. A. (1974b) Chimeric Drosophila adults produced by transplantation of nuclei into specific regions of fertilized eggs. Dev. Biol. 39, 286294.

Raff, J. W., and Glover, D. M. (1989) Centrosomes, and not nuclei, initiate pole cell formation in Drosophila embryos. Cell 57, 611-619.

Schüpbach, T., and Wieschaus, E. (1986) Maternal-effect mutations altering the anterior-posterior pattern of the Drosophila embryo. Roux's Arch. Dev. Biol. 195, 302-313.

Schüpbach, T., and Wieschaus, E. (1989) Female sterile mutations on the second chromosome of Drosophila melanogaster. I. Maternal effect mutations. Genetics 121, 101-117.

Sonnenblick, B. P. (1959) The early embryology of Drosophila melanogaster. In: Biology of Drosophila (ed.: M. Demerec), pp. 62-167. John Wiley \& Sons Inc., New York.

Spradling, A. C., and Rubin, G. M. (1982) Transposition of cloned P elements into Drosophila germline chromosomes. Science 218, 341-347.

Spurway, H. (1948) Genetics and cytology of Drosophila subobscura: IV. An extreme delay in gene action causing sterility. J. Genet. 49, 126-140.

Strome, S., and Wood, W. B. (1983) Generation of asymmetry and segregation of germ-like granules in early Caenorhabditis elegans. Cell 35, 15-25.

Technau, G. M., and Campos-Ortega, J. A. (1986) Lineage analysis of transplanted individual cells in embryos of Drosophila melanogaster. Wilhelm Roux's Arch. Dev. Biol. 195, 489-498.

Togashi, S., and Okada, M. (1983) Effects of UV-irradiation at various wavelengths on sterilizing Drosophila embryos. Dev. Growth Differ. 25, 133-141.

Togashi, S., Kobayashi, S., and Okada, M. (1986) Functions of maternal mRNA as a cytoplasmic factor responsible for pole cell formation in Drosophila embryos. Dev. Biol. 118, 352360 .

Ueda, R., and Okada, M. (1982) Induction of pole cells in sterilized Drosophila embryos by injection of subcellular fraction from eggs. Proc. Natl. Acad. Sci. USA 79, 6946-6950.

Wang, C., Dickinson, L. K., and Lehmann, R. (1994) Genetics of nanos localization in Drosophila. Dev. Dyn. 199, 103-115.

Wylie, C. C., Heasman, J., Snape, A., O'Drisco, M., and Holwill, S. (1985) Primordial germ cells of Xenopus laevis are not irreversibly determined early in development. Dev. Biol. 112, 6672.

Yamazaki, Y., and Okada, M. (1989) Differences in fine structure between normal and RNA-induced Drosophila pole cells. Dev. Growth Differ. 31, 549-556. 